\title{
Technical issues surrounding the preparation, characterisation and testing of nanoparticles for ecotoxicological studies
}

\author{
R. Tantra, S. Jing \& D. Gohil \\ National Physical Laboratory, UK
}

\begin{abstract}
There is a genuine concern about how engineered nanoparticles affect the environment and this has resulted in a detailed two-part study that is presented in this paper. The first part of the study investigates some of the issues surrounding the dispersion and characterisation of nanoparticle suspensions, which are critical in order to carry out testing for understanding the ecotoxicological properties of nanoparticles in the environment. Cerium oxide $\left(\mathrm{CeO}_{2}\right)$ nanoparticles were dispersed in de-ionised (DI) water and subsequently characterised using Dynamic Light Scattering and Scanning Electron Microscopy. Results showed that the reliability of data obtained depended heavily on the need to control the dispersion step and to understand limitations associated with current measurement techniques. The second part of the study investigated the fate of nanoparticles when dispersed in three different ecotox media (seawater compared with media of fish and Daphnia), in an attempt to identify initial measurement concerns. Visual sedimentation experiments showed that nanoparticles (within a two day period) were relatively unstable in these ecotox media (relative if dispersion was carried out in DI water). Although most particles aggregated into larger clusters, SEM images showed the presence of nanosize clusters $(<800 \mathrm{~nm})$, which were still present in these media. It is the presence of these nanosize particles that will be of utmost concern, if the hypothesis that relates particle size and toxicological activity holds true.
\end{abstract}

Keywords: nanoparticles, aquatic ecotoxicity, characterisation, dispersion, aggregation, agglomerates, DLS, SEM. 


\section{Introduction}

Over the past few years, research concerning nanoparticle toxicity has attracted public concern [1]. Particularly, in assessing their toxicological significance, several studies $[2,3]$ indicated that nanoparticle toxicity was governed by their small size and high surface area, which subsequently would lead to greater chemical reactivity. In an attempt to address this public concern, the OECD (Organization for Economic Co-operation and Development) has recently launched a sponsorship program on nanoparticle safety assessment that requires global co-operation. This has resulted in the United Kingdom to launch the PROPEcT LINK project, which aims to fulfil the UK's contribution for the testing of zinc oxide $(\mathrm{ZnO})$ and cerium oxide $\left(\mathrm{CeO}_{2}\right)$ nanoparticles.

Central to toxicological investigation of nanoparticle is the need to link toxicological activity with physicochemical properties [4]. In other words, what are the physical/chemical parameters of the nanoparticles that are most responsible for toxicological activity? This type of research has been conducted in the past but the conclusions drawn from such studies are often contradictory in nature, suggesting the need to successfully develop and establish internationally agreed standardised protocols. For example, the toxicity of carbon nanotubes has been one of the most pressing questions in nanotechnology [5]. Recently, Poland and co-workers [6] have shown experimentally that nanotubes display similar toxic responses to asbestos fibres, whereas Koyama et al. [7] have reported that the extent of toxicity of carbon nanotubes was low if compared to asbestos.

The purpose of the present study was to fulfil two objectives. The first objective concerns issues surrounding development of protocols. This research explores some of the issues associated with dispersing and subsequently characterising $\mathrm{CeO}_{2}$ in DI water; DI water was used as past results showed good stability when nanoparticles were dispersed in such media as indicated by their high zeta-potential values $[8,9]$. Results showing the effect of using different deagglomeration tools to disperse the nanoparticles are presented in this paper. In the development of characterisation protocols, the importance of understanding the limitations of the technique (particularly "limit of quantification") for the intended use will be evaluated. For example, as nanotoxicological investigations often require the need to conduct analysis at extremely low particle concentrations, $\sim$ nanogram per litre $(\mathrm{ng} / \mathrm{L})$ or less [10], the effects on data acquired (from various techniques: Dynamic Light Scattering (DLS) for particle size and zeta-potential measurement, and scanning electron microscopy (SEM)) upon dilution of nanoparticle concentration has been investigated. A technology "space map" is presented to show the limitations associated with using these tools.

The second objective of this work was to determine the fate of the nanoparticles in ecotox media and to identify any initial concerns. According to hypothesis, it is aspects of particle size characteristics that dominate the toxic profile of nanoparticles [11]. It would be expected that at high salt concentrations, the ecotox media would result in particle instability upon dispersion resulting in the formation of large aggregates/agglomerates that would 
eventually sediment out [12] and reduce toxicity. The central question here is: Will all of the nanoparticles sediment out? To assess this, dispersions of $\mathrm{CeO}_{2}$ and $\mathrm{ZnO}$ were prepared in three different ecotox media (seawater, media of fish and Daphnia) and results from a visual sedimentation type experiment will be presented here. Also, results will be compared relative to dispersions in DI water. SEM analysis was used to characterise nanoparticles and agglomerates in dispersions after a two-day hold period.

\section{Experimental}

\subsection{Materials and sample preparation}

Z-Cote Zinc Oxide ( $\mathrm{ZnO}$ with a nominal particle diameter size of $100 \mathrm{~nm}$ ) and Nanograin $\left(\mathrm{CeO}_{2}\right.$, with an average particle size of $\left.\sim 50-70 \mathrm{~nm}\right)$ were supplied by BASF SE and Umicore Belgium, respectively. Nanoparticles were dispersed using the protocol below, in one of four possible aqueous liquid media: deionised (DI) water and three ecotox media (seawater and media of fish and Daphnia). DI water from Millipore, MilliQ system was used to prepare all aqueous solutions and suspensions. Ecotox media was prepared as follows: a) Seawater - 25 g per L of Tropic Marine Sea Salt (Tropical and Marine Limited), was prepared, $\mathrm{pH} \sim 7.5$. b) Daphnia freshwater media. Salts (196 mg $\mathrm{CaCl}_{2} \cdot 2 \mathrm{H}_{2} \mathrm{O}, 82 \mathrm{mg} \mathrm{MgSO}_{4} \cdot 7 \mathrm{H}_{2} \mathrm{O}, 65 \mathrm{mg} \mathrm{NaHCO} 3,0.002 \mathrm{mg} \mathrm{Na}_{2} \mathrm{SeO}_{3}$ (as obtained by appropriate dilutions of a $2 \mathrm{mg} / \mathrm{ml}$ stock solution) were dissolved in $1 \mathrm{~L}$ of DIwater. Upon continued stirring, DI water was further added so that the final $\mathrm{pH} \sim 7.5$ and conductivity was between $\sim 360-480 \mu \mathrm{S} / \mathrm{cm}$. End volume $\sim 1-$ 1.5 L. c) Fish freshwater media. This was prepared in three separate steps. First, salts $\left(11.76 \mathrm{~g} \mathrm{CaCl}_{2} \cdot 2 \mathrm{H} 2 \mathrm{O}, 4.93 \mathrm{~g} \mathrm{MgSO}_{4} \cdot 7 \mathrm{H}_{2} \mathrm{O}, 2.59 \mathrm{~g} \mathrm{NaHCO}_{3}, 0.23 \mathrm{~g} \mathrm{KCl}\right)$ were dissolved separately in $1 \mathrm{~L}$ of DI water to make four separate stock solutions. Second, $25 \mathrm{~mL}$ of each salt stock solution was aliquoted into a clean bottle and diluted in DI water (made up to $1 \mathrm{~L}$ volume). Third, $200 \mathrm{ml}$ of the stock solution from Step 2 was aliquoted and further diluted with DI water (made up to $1 \mathrm{~L}$ volume). For long-term storage, these ecotox solutions were autoclaved and kept refrigerated until needed.

\subsection{Nanoparticle dispersion in aqueous liquid media}

Nanoparticle powders were weighed into small clean vials using an analytical mass balance. To disperse, a few drops of the appropriate liquid media were added to the vial and mixed into a thick paste using a spatula. $\sim 15 \mathrm{~mL}$ of liquid media was then added to the paste and the whole mixture gently stirred using a spatula. De-agglomeration step was then carried out and this is very much dependent on which de-agglomeration tools were employed. If an ultrasonic probe (130 Watt Ultrasonic Processors) was used, the ultrasonic probe tip (6 $\mathrm{mm}$ Ti) was inserted half way down the $15 \mathrm{ml}$ volume of dispersed nanoparticles and sonicated with $90 \%$ amplitude for $20 \mathrm{~s}$ (unless otherwise stated); temperature measurements were made using a digital thermometer (Fisher Scientific) before 
and after the sonication step. After sonication, the nanoparticle suspension was diluted using the appropriate liquid media, in order to make up to $1 \mathrm{~L}$ total volume (unless otherwise stated), a glass rod was used to gently mix the final dispersion, to ensure homogeneity. If other de-agglomeration tools were used instead (PowerGen Fisherbrand 500 homogeniser or Kinematica Overhead stirrer PX-SR 90 D), then the initial $15 \mathrm{ml}$ dispersion mixture was diluted straightaway into the appropriate liquid media, to make up a total $1 \mathrm{~L}$ in volume. The homogeniser or overhead stirrer was lowered into the dispersion and operated at a constant speed for 1 minute to create maximum vortex action without spillage in a $1 \mathrm{~L}$ beaker.

For the purpose of investigating "limit of quantification", $\mathrm{CeO}_{2}$ was dispersed in DI water using an ultrasonic probe. A stock solution of $500 \mathrm{mg} / \mathrm{L}$ was prepared and appropriate dilutions with DI water were made from this stock. For the purpose of "visual sedimentation" tests, eight separate nanoparticle suspensions $(500 \mathrm{mg} / \mathrm{L})$ were prepared $\left(\mathrm{ZnO}\right.$ and $\mathrm{CeO}_{2}$ dispersed separately in DI water and three ecotox media) in media bottles. Optical images showing the state of the dispersion in the bottles were recorded using a digital camera at set intervals over a period of two days. In between recording the images, the bottles were stored in the dark.

\subsection{DLS (particle size and zeta-potential) analysis}

The instrument employed for particle size analysis and zeta-potential measurements was a Zetasizer Nano ZS (Malvern Instruments, UK) with $633 \mathrm{~nm}$ red laser. The same instrument is also able to make zeta-potential measurements, by using a laser Doppler electrophoresis configuration. Detailed protocols for DLS particle size and zeta-potential measurements have been reported elsewhere [8].

\subsection{Scanning electron microscopy (SEM) analysis}

Scanning electron microscope images were recorded using a Carl Zeiss Supra 40 field emission scanning electron microscope, in which the optimal spatial resolution of the microscope was a few nanometres. In-Lens detector images were acquired at an accelerating voltage of $15 \mathrm{kV}$, working distance of $\sim 3 \mathrm{~mm}$, tilt angle $0^{\circ}$. For analysis of the "as received" nanoparticle powder, a small scoop of the nanoparticle powder was sprinkled over an SEM carbon adhesive discs; one side of the carbon disc was placed securely on a metal stub, whilst the other side was exposed to the nanoparticle powder. Excess powder on top of the disc was removed by gently tapping the stub on its side until an even (light) coating of powder on the surface was apparent. Detailed protocols associated with sample preparation (of depositing nanoparticles dispersed in liquid media on to poly-L-lysine slides) suitable for SEM analysis have been reported elsewhere [8]. 


\section{Results and discussion}

\subsection{Nanoparticle dispersion}

SEM images of the "as received" for $\mathrm{ZnO}$ and $\mathrm{CeO}_{2}$ nanoparticles are given in Figure 1a) and show that polydispersity for both particle size and shape is high. These SEM images also show evidence of extensive aggregation and agglomeration (fusion of particles) that exists in both nanoparticles; this is particularly evident in $\mathrm{CeO}_{2}$.
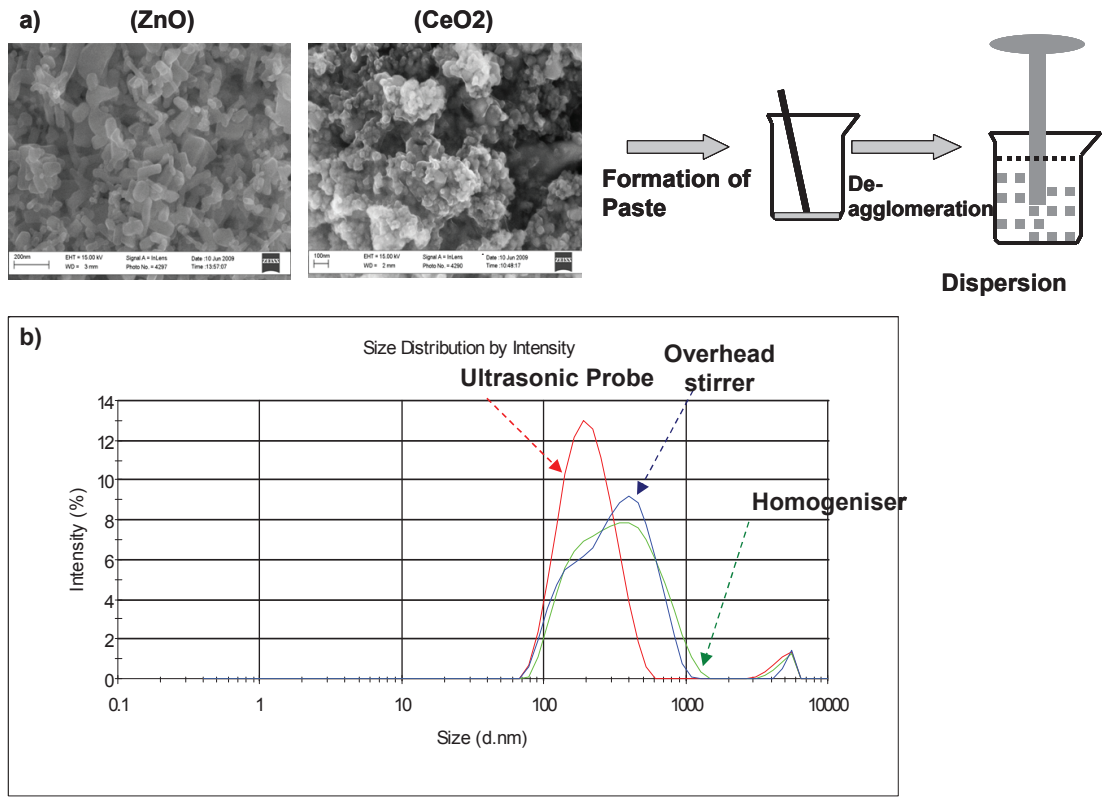

Figure 1: Nanoparticle dispersion in aqueous media: a) a schematic of the dispersion step from the "as received" powders (SEM images shown; scale bars $200 \mathrm{~nm}$ for $\mathrm{ZnO}$ and $100 \mathrm{~nm}$ for $\mathrm{CeO}_{2}$ ) b) Particle size distribution of $\mathrm{CeO}_{2}$ in DI water $(50 \mathrm{mg} / \mathrm{L})$ and the effects of using different de-agglomeration tools at an exposure time of 1 minute).

Figure 1a) shows a schematic illustrating the steps of the dispersion protocol, as detailed in the Experimental section. Overall, this involved two essentials steps: a) the wetting of the nanoparticle powder into a paste, so as to substitute solid air-interfaces with solid-liquid interfaces, as recommended by ISO 14887: 2000 [13] b) de-agglomeration of nanoparticles using an appropriate tool, so as to introduce sufficient shear energy such that aggregates/agglomerates are broken down using an appropriate de-agglomeration tool, ideally to individual primary particles [14]. Figure 1b) shows the particle size distribution (by 
intensity as reported by DLS) of $\mathrm{CeO}_{2}(50 \mathrm{mg} / \mathrm{L})$ in DI water, when dispersed using an ultrasonic probe, with an exposure time of 1 minute. Results show a particle size distribution between $68-615 \mathrm{~nm}$ in size. The plot also shows the effect of altering the dispersion protocol step, when either an overhead stirrer or homogeniser was employed instead of ultrasonic probe. Results indicate a much broader particle size distribution, with particle sizes as big as $1 \mu \mathrm{m}$; the much bigger size particles found in the dispersions using these tools can only be explained by the much lower shear energy provided (to result in insufficient deagglomeration/de-aggregation) if compared to the ultrasonic probe. Undoubtedly the final stability after the dispersion will be governed by the inherent properties of the liquid media and their interactions with the nanoparticles [15]. Overall, due to its effectiveness in de-agglomerating, the ultrasonic probe proved to be the tool of choice for the dispersion protocol.

Another variable that can potentially affect the particle size distribution is the length of time that the dispersion is exposed to i.e. the "exposure time". Figure 2a). shows the effect of changing the "exposure time" on the mean particle size. As expected, increasing the de-agglomeration time from $5 \mathrm{~s}$ to $50 \mathrm{~s}$ resulted in a reduction of particle size; the longer the exposure time the more deagglomeration occurred resulting in smaller particle sizes. However, increasing

a)

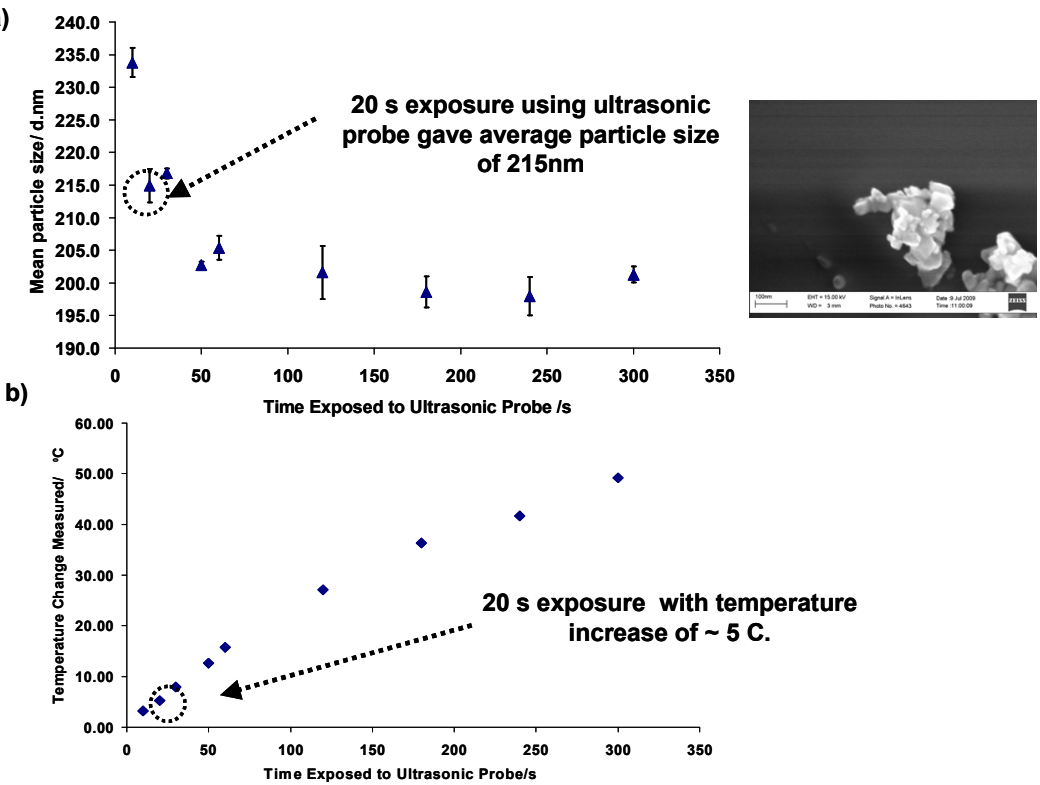

Figure 2: Effect of varying "exposure time" (using an ultrasonic probe) on the DLS mean particle size of $\mathrm{CeO}_{2}$ in DI water $(50 \mathrm{mg} / \mathrm{L})$ on: a) mean particle size (inset: corresponding SEM image, when $20 \mathrm{~s}$ exposure time was used; scale bar shown reads $100 \mathrm{~nm}$ ) and b) corresponding temperature change measured in the dispersion, after exposing the dispersion with the ultrasonic probe. 
the exposure time beyond $50 \mathrm{~s}$ does not seem to result in further breaking up of nanoparticles; it is hypothesised that shear energy provided by the ultrasonic probe was sufficient to de-aggregate but cannot sufficiently break nanoparticles that have fused together (i.e. agglomerates). A side effect of ultrasonication is the increase in temperature of the dispersion, due to the high shear energy that it provides [16]. Figure 2b) shows the change in temperature that occurred as a result of dispersion at various "exposure times" and the relationship between the two variables is approximately linear. Ideally, temperature changes in the dispersion should be minimised and so, a 20 s exposure time was chosen for the dispersion protocol as this gave $\sim 5^{\circ} \mathrm{C}$ temperature increase (with corresponding particle size of $215 \mathrm{~nm}$ as shown in the DLS and the corresponding SEM image in Figure 2a). Results so far have shown the importance of having well controlled protocols for dispersions, as slight changes to procedures can potentially influence the particle size distribution of the resultant dispersion.

\subsection{Limitations of characterisation tools}

Figure 3a) and b) shows the effect of reducing $\mathrm{CeO}_{2}$ nanoparticle concentration in DI water (from $500 \mathrm{mg} / \mathrm{L}$ to $0.001 \mathrm{mg} / \mathrm{L}$ ) on DLS mean particle size and zetapotential, respectively. Both plots show that values measured are similar above certain concentration. However, under extreme dilution conditions, data values shift significantly, yielding what is thought to be erroneous results. The erroneous data defines the limit of quantification for particle size and zetapotential to be 0.1 and $50 \mathrm{mg} / \mathrm{L}$, respectively. The results at extreme dilution is not surprising and explanations have been previously attributed: the inherent homodyne configuration of the optics a combination of: increase in signal contribution due to extraneous particles and inherent sensitivity of the detector, which defines the limit of quantification for particle size and zeta-potential, respectively [17]. Figure 3c) shows a series of SEM images of $\mathrm{CeO}_{2}$ (dispersed in DI water and subsequently adsorbed on the surface of poly-L-lysine substrates), upon changing nanoparticle concentration within the dispersion. It is apparent that the particle size distribution changes dramatically when nanoparticle concentration in the dispersion is diluted from $500 \mathrm{mg} / \mathrm{L}$ to $10 \mathrm{mg} / \mathrm{L}$. A much-reduced number of nanoparticles and a tendency for smaller particles adhering to the surface were observed under the extreme dilution conditions.

Unlike the DLS instrument, the limit of quantification is governed by the adsorption kinetics of the nanoparticles on to the substrates during the sample preparation step. At low nanoparticle concentrations, it is the diffusion rate of the particle that will dominate and this in turn explains why the smaller particles are preferentially adsorbed [12]. Overall, identifying the limit of quantification for an individual analytical procedure is important, so as to understand when data becomes unreliable. This is particularly of importance to nanoecotoxicological investigations as researchers in this field are often interested in making measurements under extreme dilute conditions, in the order of less than a few $\mathrm{ng} / \mathrm{L}[10]$. 


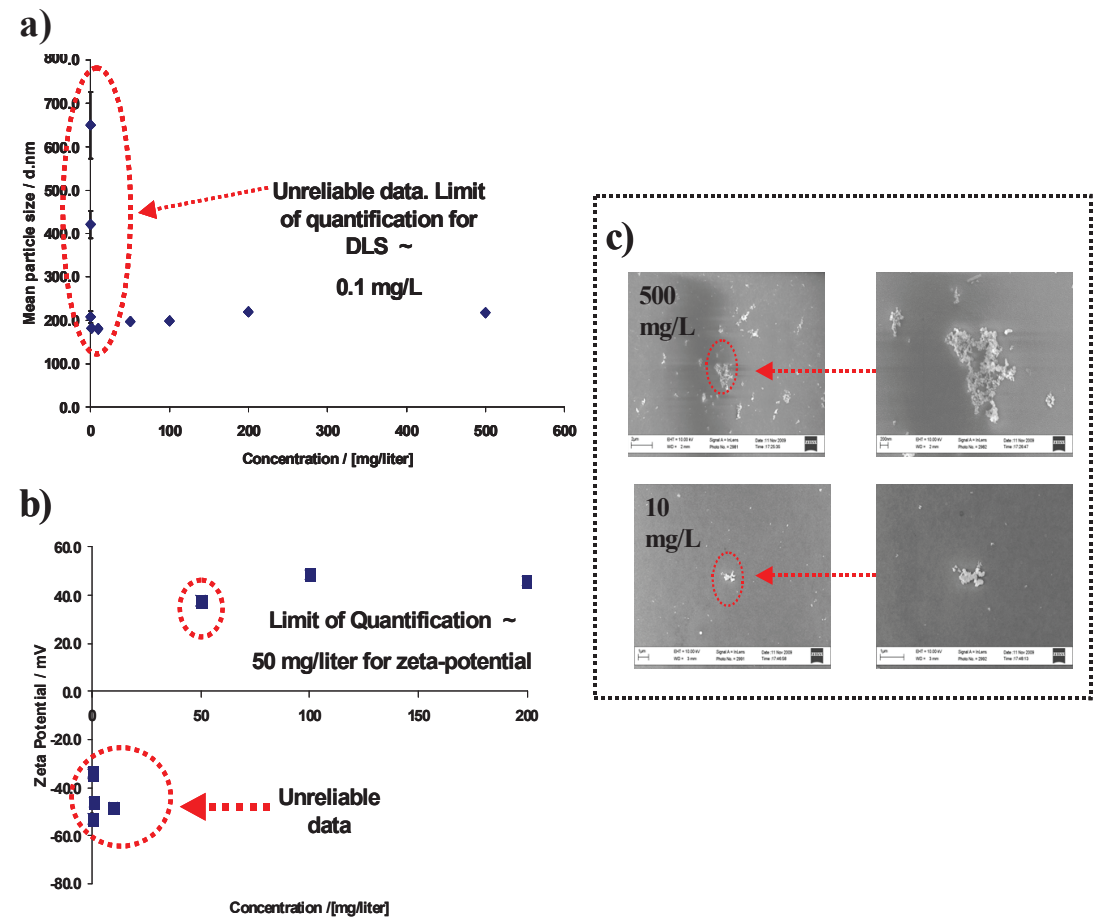

Figure 3: $\mathrm{CeO}_{2}$ dispersed in DI water and the effect of varying nanoparticle concentration on: a) DLS mean particle size b) zeta-potential c) SEM of nanoparticles adsorbed on poly-L-lysine slides. Scale bars SEM: for $500 \mathrm{mg} / \mathrm{L}$ (reads $2 \mu \mathrm{m}$ and $200 \mathrm{~nm}$, for low and high magnification, respectively) and for $10 \mathrm{mg} / \mathrm{L}$ (reads $1 \mu \mathrm{m}$ for both).

Figure 4 aims to identify where common laboratory techniques like DLS and SEM sit on the technology space map. Three important criteria have been identified as being essential: sensitivity (x-axis), selectivity (y-axis) and representativeness (z-axis). Ideally, an instrument should have a high degree of sensitivity (to single particle level), high selectivity (to measure in the presence of potentially interfering substances in the ecotox media [10] and high representativeness (such that the data is a representation of the entire population rather than a subset; this will subsequently contribute towards the accuracy and repeatability of the measurements); the ideal tool sits on vertex $\mathrm{C}$. According to the technology road map, the DLS and SEM sit on vertex A and B, respectively. The DLS, belongs to a category of "population based methods"; they may not have the desired combination of high sensensitivity and selectivity but data are highly representative of the entire population. SEM on the other hand, belongs to a category of "single particle based methods", which are highly sensitive and selective but data obtained lacks "representativeness". Future research activities should therefore employ and subsequently validate a technique that belongs on vertex $\mathrm{C}$ on this space map. 


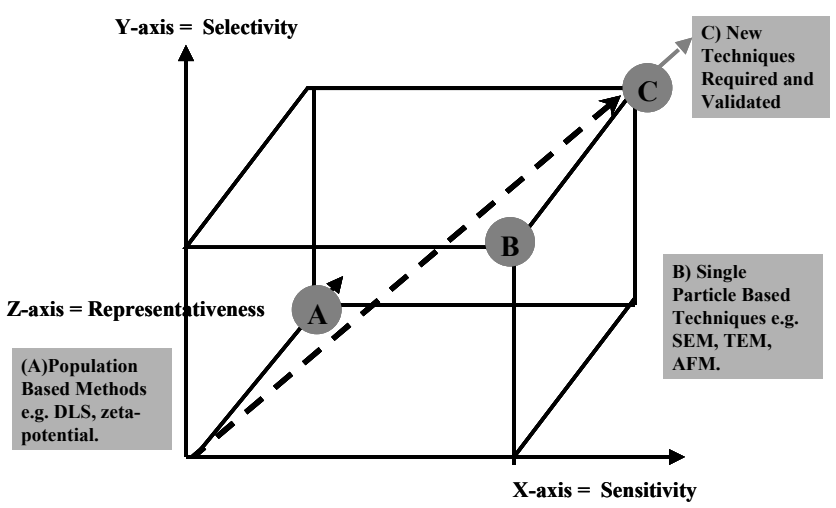

Figure 4: Technology space map: tools/techniques for nanoparticle characterisation in complex environmental media assessed against three identified criteria of: sensitivity (x-axis), selectivity (y-axis) and "representativeness" (z-axis).

\subsection{Visual sedimentation}

Figure 5 shows a typical result from the visual sedimentation experiment of when nanoparticles (either $\mathrm{ZnO}$ or $\mathrm{CeO}_{2}$ ) are dispersed in one of the ecotox media $(500 \mathrm{mg} / \mathrm{L})$. After two days, the dispersion inside the bottle showed complete sedimentation, leaving a clear solution above the sediment. Interestingly, if nanoparticles were dispersed in DI water instead, a different result is apparent in that a cloudy suspension was still observed on Day 2. This suggested that the particles were more stable in DI water and subsequently a much slower sedimentation rate was observed. When salts were present in the ecotox media, this caused particle instability and resulted in aggregation/agglomeration [18]. The addition of salts in the ecotox liquid formulation employed in this study was sufficient to cause particle aggregation, resulting in a much faster sedimentation rate. If such sedimentation events occurred in ecotoxicological relevant media, then this raises the question as to whether there would be significant safety concerns. On Day 3, samples inside the bottles were analysed with an SEM; most particles present in the dispersion were shown to constitute largely of micron-sized particles, as expected. However, upon careful examination, some smaller clusters of both nanoparticles (particle diameter size of less than $\sim 800 \mathrm{~nm}$ ) were present in all four media, as shown in Figure 5; particle diameter size was estimated by assuming spherical shape cluster. From the SEM examination, this was the case for $\mathrm{ZnO}$ disperse in DI water, seawater and Daphnia liquid media; for the case of $\mathrm{CeO}_{2}$, this was true upon dispersion in DI water and fish media. If the hypothesis that relates particle size with toxicity holds true, then it would be the presence of such nanosize clusters that should be of utmost concern to the aquatic environment. 


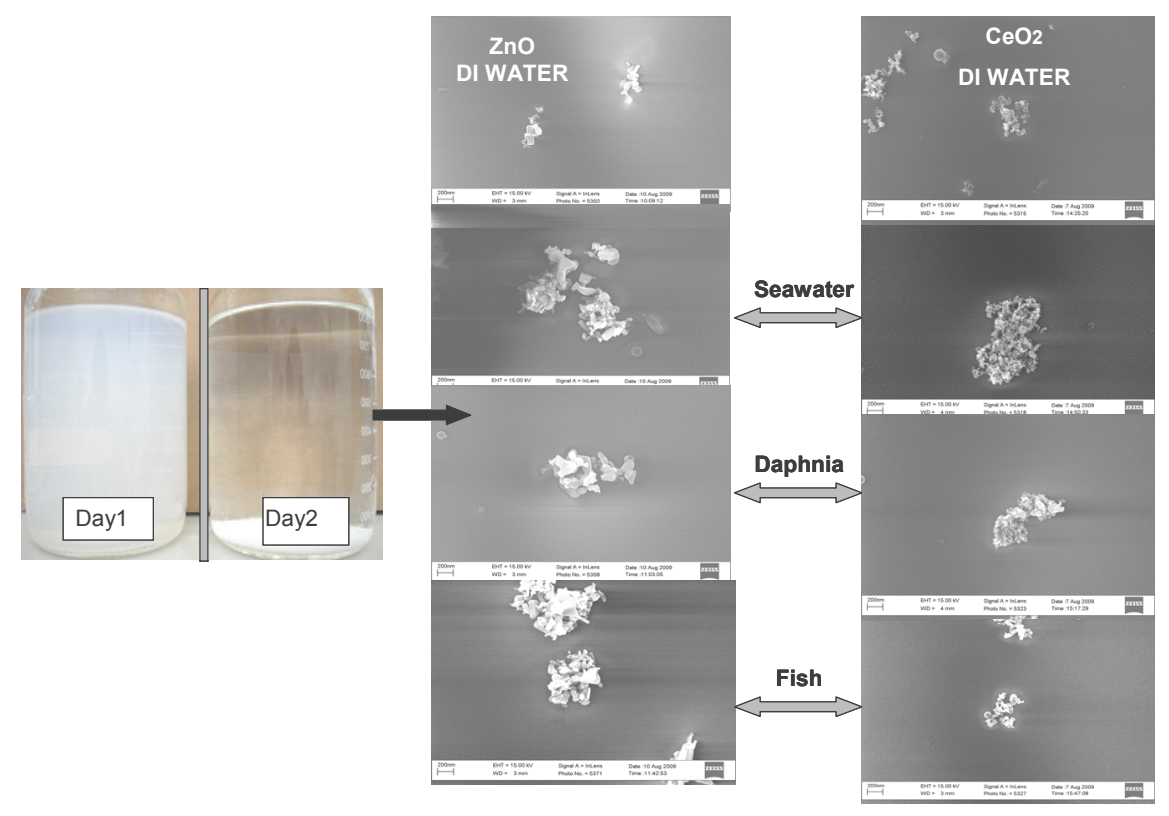

Figure 5: Visual sedimentation experiment of nanoparticles (typical of either $\mathrm{ZnO}$ or $\mathrm{CeO}_{2}$ dispersed in an ecotox media (seawater or daphnia or fish media), during a two-day period. SEM images show the presence of nanosize clusters of nanoparticles present in the bottles for both $\mathrm{ZnO}$ and $\mathrm{CeO}_{2}$ (obtained on third day); scale bar on all SEM reads $200 \mathrm{~nm}$.

\section{Conclusion}

While most nanoparticles were shown to aggregate out of solution when immersed in ecotoxicological media, some nanosized clusters were still present. If this was to occur in a real environmental setting, then there is potential for aquatic organisms to ingest such small particles. Through time, these small particles can accumulate and it is the accumulation of dose that can subsequently formulate a problem. In terms of protocol developments in ecotoxicological investigations, there is a need to:

a) have well controlled, agreed protocols on dispersion and characterisation

b) identify, develop and validate suitable tools / technology that can offer a combination of high sensitivity, selectivity and "representativeness"

\section{Acknowledgements}

This work is financed under Defra PROSPEcT LINK project. The authors would like to thank Mr. Jordan Tompkins for initial handling and distribution of the nanoparticles; Neil Harrison and Alex Shard for helpful comments and encouragement. 


\section{References}

[1] Maynard, A.D., Pui, D.Y.H., Nanotechnology and occupational health: New technologies - new challenges. Journal of Nanoparticle Research, 2007. 9(1): p. 1-3.

[2] Oberdorster, G., Stone, V., Donaldson, K., Toxicology of nanoparticles: A historical perspective. Nanotoxicology, 2007. 1(1): p. 2-25.

[3] Nel, A., Xia, T., Madler, L., Li, N., Toxic potential of materials at the nanolevel. Science, 2006. 311(5761): p. 622-627.

[4] Durnev, A.D., Toxicology of nanoparticles. Bulletin of Experimental Biology and Medicine, 2008. 145(1): p. 72-74.

[5] Jaurand, M.C.F., Renier, A., Daubriac, J., Mesothelioma: Do asbestos and carbon nanotubes pose the same health risk? Particle and Fibre Toxicology, 2009. 6: p. 14.

[6] Poland, C.A., Duffin, R., Kinloch, I., Maynard, A., Wallace, W. A. H., Seaton, A., Stone, V., Brown, S., MacNee, W., Donaldson, K., Carbon nanotubes introduced into the abdominal cavity of mice show asbestos-like pathogenicity in a pilot study. Nature Nanotechnology, 2008. 3(7): p. 423428.

[7] Koyama, S., Endo, M., Kim, Y. A., Hayashi, T., Yanagisawa, T., Osaka, K., Koyama, H., Haniu, H., Kuroiwa, N., Role of systemic T-cells and histopathological aspects after subcutaneous implantation of various carbon nanotubes in mice. Carbon, 2006. 44(6): p. 1079-1092.

[8] Tantra, R., Tompkins, J. and Quincey P., Characterisation of the deagglomeration effects of bovine serum albumin on nanoparticles in aqueous suspension. 2010. 75: p. 275-281.

[9] Necula, B.S., Apachitei, I., Fratila-Apachitei, L., Teodosiu, C., Duszczyk, J., Stability of nano-/microsized particles in deionized water and electroless nickel solutions. Journal of Colloid and Interface Science, 2007. 314(2): p. 514-522.

[10] Simonet, B.M., Valcarcel, M., Monitoring nanoparticles in the environment. . Anal. Bioanal. Chem., 2009. 393: p. 17-21.

[11] Lundqvist, M., Stigler, J., Elia, G., Lynch, I., Cedervall, T., Dawson, K. A., Nanoparticle size and surface properties determine the protein corona with possible implications for biological impacts. Proceedings of the National Academy of Sciences of the United States of America, 2008. 105(38): p. 14265-14270.

[12] Kissa, E.E., Dispersions: characterization, testing, and measurement 1999: CRC Press.

[13] ISO 14887:2000 Sample preparation -- Dispersing procedures for powders in liquids.

[14] Rhein, L.D., Schlossman M., O'Lenick A. and Somasundaran P. (Editors) and E. 3, Surfactants in personal care products and decorative cosmetics. 2006: Publisher CRC Press.

[15] Jailani, S., Franks, G. V., Healy, T. W., Zeta-potential of nanoparticle suspensions: Effect of electrolyte concentration, particle size, and volume 
fraction. Journal of the American Ceramic Society, 2008. 91(4): p. 11411147.

[16] Kanegsberg, B., Kanegsberg, E., Handbook for critical cleaning 2001: CRC Press.

[17] Tantra, R., Schulze P. and Quincey, P., Effect of Nanoparticle Concentration on Zeta-Potential Meausurement Results and Reproducibility. Particuology, in press.

[18] Murdock, R.C., Braydich-Stolle, L., Schrand, A. M., Schlager, J. J., Hussain, S. M., Characterization of nanomaterial dispersion in solution prior to In vitro exposure using dynamic light scattering technique. Toxicological Sciences, 2008. 101(2): p. 239-253. 